\title{
RADIOGENIC NEOPLASIA IN THYROID AND MAMMARY CLONOGENS
}

\section{Progress Report}

January 1, 1991 - December 31, 1991

\author{
Kelly H. Clifton \\ Department of Human Oncology \\ University of Wisconsin Comprehensive Cancer Center \\ 600 Highland Avenue \\ Madison, WI 53792
}

May 31, 1991

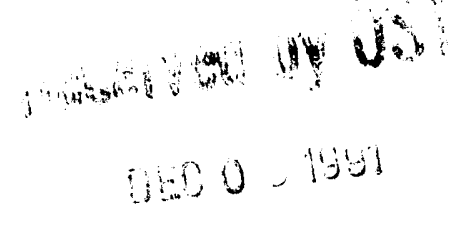

Prepared for

THE U.S. DEPARTMENT OF ENERGY

Agreement No. DE-FG02-87ER60507

N O T I C E

This report was prepared as an account of work sponsored by the United Staies Government. Neither the United States nor the Department of Energy, nor any of their employees, nor any of their contractors, subcontractors, or their employees, makes any warranty, express or implied, or assumes any legal liability of responsibility for the accuracy, completeness, or usefulness of any information, apparatus, product or process disclosed or represents that its use would not infringe privately owned rights.

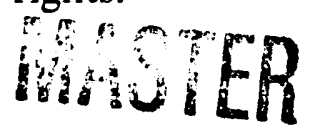




\title{
PROGRESS REPORT
}

\section{January $1991-31$ July 1991}

\begin{abstract}
We have developed rat thyroid and mammary clonogen transplantation systems for the stuci:y of radiogenic cancer induction at the target ceil level in vivo. The epithelial cell populations of both glands contain small subpopulations of cells which are capable of giving rise to monoclonal glandular structures when transplanted and stimulated with appropriate hormones. Our results before the current grant term indicated that these clonogens are the precursor cells of radiogenic cancer, and that initiation, the first step in carcinogenesis, is a common event at the clonogenic cell level. Detailed information on the physiologic control of clonogen proliferation, differentiation, and total riumbers is thus essential to an understanding of the carcinogenic process. Furthermore, knowledge of any endocrine interplay between follicles that develop from grafted thyrnid clonogens and the hypothalamic-hypophyseal system is important in interpretation of neoplastic promotion/progression of radiation-initiated cells in such grafts. During the end of the last grant year and the first half of the current grant year, we have completed analyses and summarized for publication: a) investigations on the relationship between grafted thyroid cell number and the rapidity and degree of reestablishment of the thyroid-hypothalamicpituitary axis in thyroidectomized rats maintained on a normal diet or an iodine deficient diet; b) studies of the persistence of, and the differentiation potential and functional cha acteristics of, the TSH- (thyrotropin-) responsive sub-population of clonogens during goitrogenesis, the plateau-phase of goiter growth, and goiter involution; c) studies of changes in the size of the clonogen sub-population during goitrogenesis, goiter involution and the response to goitrogen rechallenge; and d) the results of the large carcinogenesis experiment on the nature of the grafted thyroid cell number-dependent suppression of promotion/progression to neoplasia in grafts of radiation-initiated thyroid cells. One of these manuscripts has been published (Do90; Appendix I), a second is in press (Gr91a; revised, Appendix II), and two are being submitted (Gr91b; Appendix III; Do91; revised, Appendix IV). We are testing new techniques for the culture, cytofluorescent analysis and characterization mammary epithelial cells and of clonogens in a parallel project, and plan tc apply similar technology to the thyroid epithelial cells and clonogen population. Data from these studies will be user in the design of future carcinogenesis experiments on neoplastic initiation by high and low LET radiations and on cell interactions during the neoplastic process.
\end{abstract}

Introduction: The induction of cancer by ionizing radiation is a matter of great practical importance to the nuclear industry, to national defense, to radiological medicine and to the general public. It is increasingly apparent that carcinogenesis is one of the 
leading, if not the principal, dose-limiting effect of radiation exposure (Co90). Quantitative information at the cellular level is essential to an understanding of the mechanisms of radiogenic neoplastic initiation and the stages of promotion and progression to overt neoplasia. We have developed two experimental models, the rat thyroid and rat mamrnary clonogen transplant systems, for the quantitative study of radiation carcinogenesis at the cellular level in vivo. The ideal model for such studies would allow measurement of: a) the initial number of target cells at risk in vivo; b) the number of such cells which survive radiation exposure; and c) the fraction of surviving target cells which have suffered radiogenic neoplastic initiation ( $\mathrm{Cl} 80)$. The latter currently requires that each initiated cell give rise to an overt neoplasm.

Experiments with these model systems completed before the current grant period showed a) that both rat mammary and thyroid glands contain subpopulations of clonogenic epithelial cells which when transplanted and stimulared with appropriate hormones give rise to functional monoclonal glandular structures, b) that these clonogeus are the progenitor cells of radiogenic thyroid and mammary cancer, c) that such cells are capable of a high degree of intercellular repair when left in a tissue environment after irradiation before removal for transplantation assay, d) that at the target clonogen level, radiogenic initiation, the first step in the carcinogenic process, is a common event, and e) that the promotion/progression of initiated thyroid clonogens to overt cancer is suppressed by the addition of unirradiated thyroid cells to the transplant inoculum $(\mathrm{Cl} 89, \mathrm{Cl} 90)$. The thyroid clonogens have many of the characteristics of stem cells (C191).

As noted in the Abstract above, much of the end of the last year and the start of the current grant year has been devoted to preparation for publication of the data from studies of the control of the clonogen sub-population and its role in thyroid physiology and hyperplasia, and in completion and analysis of the large carcinogenesis experiment on cellcell interactions during neoplastic promotion/progression in grafts of radiation-initiated thiyroid cells. We have also begun new studies on the culture of clonogenic and nonclonogenic thyrocytes, and on the identification and sorting of cell sub-populations by cell surface antigens and fluorescence activated cell sorting, and are planning experiments on the persistence of cionogenic cells in grafts after transplantation. Progress in these areas is described below.

Dependence of reestablishment of the thyroid-hypothalamic-pituitary axis in thyroidectomized rats on the number of grafted thyroid cells, and on iodine intake: Groups of thyroidectomized rats were each sham grafted or grafted with $2.4 \times 10^{3}$ to $3.1 \mathrm{~J} \times 10^{5}$ syngeneic rat thyroid cells. The grafted rats and intact ungrafted controls were then fed either a normal diet or a diet deficient in iodine. Blood samples were collected at weekly intervals for a ven weeks, and the serum concentrations of $T_{3}$ (triiodothyronine), $T_{4}$ (ihyroxine) and TSH were determined by radioimmunoassay. The results show the progressive restoration 
of hormone levels toward the norm in the thyroidectomized animals fed a normal diet and grafted with the largest cell numbers; those which received fewer grafted cells had progressively lesser and slower restoration. In intact ungrafted rats fed iodine deficient diets there was selective preservation of $T_{3}$ secretion. In thyroidectomized rats fed the low iodine diet and grafted with the highest thyroid cell doses, $T_{3}$ levels returned to near normal more rapidly than the other hormones. This selective hormone secretion pattern would have potential adaptive value under conditions of limited iodine availability. The details of these experiments were discussed in our last Progress Report and are now published (Do90; Appendix I).

Thyroid clonogen proliferative and differentiative potentials during goitrogenesis and goiter involution: We have measured the concentrations of clonogens within the cell populations of thyroid glands by their capacity to give rise to monoclonal multicellular structures (FU, follicular units) at the sites of transplantation of known numbers of monodispersed thyroid cells in thyroidectomized recipient rats. The mean number of thyroid cells per graft site required to produce at least one FU in 50\% of the sites (the FD50, follicle dose-50\%) in such animals is an inverse function of the clonogenic fraction (Cl80, $\mathrm{Cl}$ 190); these two parameters are used to express clonogen concentrations. The formation of FU is, we believe, maximized by exposure of the clonogens to the elevated TSH levels consequent to thyroidectomy-induced thyroid hormone deficiency in such grafted rats. In similar grafts in intact euthyroid recipients, i.e. in the presence of normal TSH leveis, about one fourth to one sixth as many of the grafted clonogens give rise to FU as in thyroidectomized rats.

As noted in our last Progress Report, the FD50 assay system was used to quantitate changes in the clonogenic fractions of thyroid cells during goitrous growth, in quiescent goiters and during goiter involution. These data have now been analyzed and are currently in press (Gr91a; Appendix II). Goiters were induced either by aminotriazole treatment of rats given a normal diet (ATA/ND) or by perchlorate treatment of rats given a low iodine $\operatorname{diet}\left(\mathrm{KClO}_{4} / \mathrm{LID}\right)$. Rapid goitrous growth occured during the first six weeks of treatment after which gland growth plateaued. TSH levels peaked at about the same level at six weeks of treatment with the two regimens and remained elevated as long as the goitrogens were administered. Plateau-phase goiters induced by ATA/ND were more than twice as large as those induced by $\mathrm{KClO}_{4} / \mathrm{LID}$.

Importantly, suspensions of cells prepared from either goiter type contained significant numbers of clonogenic cells as measured by transplantation and FU formation. This was true despite the fact that the clonogenic fractions of goiter cell preparations were significantly less than those of untreated control glands. Furthermore, the clonogenic fractions of cells from ATA/ND goiters were significantly greater than those of $\mathrm{KClO}_{4} / \mathrm{LID}$ goiters. When transplanted to recipient rats, the clonogens that persisted in both goiter 
types responded to TSH stimulation in a fashion indistinguishable from that of clonogens from untreated glands. For example, the efficiency of FU formation in grafts of suspensions of goiter cells and of normal thyrocytes in euthyroid recipients was reduced about the same extent from that in grafts of the same cell suspensions in thyroidectomized recipients. The FU formed in the graft sites of clonogens from goiters were not detectably different morphologically or functionally from untreated clonogens (Gr91a; Appendix II).

As discussed above, studies of thyroid hormones and TSH in the sera of thyroidectomized graft recipients showed that reestablishment of thyroid function depended on the number of thyroid cells from untreated rats transplanted per recipient (Do90; Appendix I). Although this dependence was clear when the donors were euthyroid rats, this appears to be due to the consistency of the clonogenic fractions of such rats. In the current experiments, groups of thyroidectomized rats were transplanted with series of cell suspensions prepared from either of the two goiter types or from normal glands. The cell concentrations in these suspensions were chosen to contain the same numbers of thyroid clonogens; the total thyroid cell concentrations in these suspensions thus varied according to the previous treatment of the donor rats. The results show unequivocally that thyroid hormone secretion by such grafts is dependent on the numbers of clonogens grafted, irrrespective of the total numbers of thyroid cells per graft or the previous treatments of the donor rats (Gr91a; Appendix II).

Finally, FD50 assays of cell suspensions from involuting goiters show preferential preservation of the clonogen sub-population. The clonogens persist despite the massive cell loss that occurs during involution (Gr91a).

In sum, these data are highly supportive of the conclusion that goiters contain subpopulations of pre-existent stem cell-like clonogens which retain their responsiveness to TSH and their proliferative and differentiative potentials during goiter growth, the goiter growth plateau phase, and goiter involution. It is very likely that these cells are the progenitor subpopulation from which tumors arise after long term exposure to goitrogens or after radiation exposure.

Clonogenic cell populations during goitrogenesis, goiter involution and goitrogen rechallenge: A series of experiments were performed to determine the ates of the subpopulations of clonogens during initial goitrogenesis with $\mathrm{ATA} / \mathrm{ND}$ or $\mathrm{KClO}_{4} / \mathrm{LID}$ treatment, following withdrawal of goitrogens and during goitrogen rechallenge with the aim of gaining further insight into the pathogenesis of thyroid neoplasia. The clonogenic fraction of cells from untreated age-matched control thyroid glands was $5.8 \times 10^{-3}$ cells; that of ATA-NDinduced goiters was about 20 -fold smaller $\left(1.9 \times 10^{-4}\right.$ cells $)$ and of $\mathrm{KClO}_{4} / \mathrm{LID}$-induced goiters, about 60 -fold smaller $\left(9.8 \times 10^{-5}\right.$ cells $)$ than the controls. With continued ATA/ND treatment after goitrous hyperplasia had ceased, the clonogenic fraction increased an order of magnitude to $2.0 \times 10^{-3}$ cells; continued $\mathrm{KClO}_{4} / \mathrm{LID}$ treatment had little effect. 
The changes in clonogenic fractions induced by both goitrogenic regimens were reversed during involution. Rechallenge of the involuted glands with a second exposure to the goitrogens induced changes in the clonogenic fractions similar to those which occured during the first treatment period. It is important to note that the clonogenic fraction in the glands of ATA/ND-treated rats were greater than those of $\mathrm{KClO}_{4} / \mathrm{LID}$-treated rats at all time points studied despite similar TSH values.

The two goitrogenic regimens which act through different biochemical mechanisnis thus affect the goitrogenic fractions in different ways. Despite this fact, the changes in the clonogen sub-populations induced by both goitrogens are reversed following withdrawal of treatment. The behavior of the clonogenic sub-populations is consistent with that to be expected of stem cells. These data are submitted for publication (Gr91b; Appendix III).

Nature of cell-cell interactions in thyroid grafts during promotion/progression to neoplasia: In previous studies, we had observed that the incidence of carcinomas per irradiated clonogen in grafts of thyroid cells in thyroidectomized recipients maintained on a low iodine diet was inversely related to the total number of cells grafted (Mu84). Furthermore, when unirradiated cells were added to irradiated cell grafts, final carcinoma incidence was further suppressed (Wa88). We reasoned that this suppressive effect could be mediated either: a) systemically by partial reestablishment of the thyroid-hypothalamicpituitary axis by the grafted cells, the rapidity and degree of which is grafted cell numberdependent as demonstrated above (Do90; Appendix I); b) locally by direct suppressive thyroid cell:cell interactions within the grafts as reported in the tracheal epithelium by Terzaghi-Howe (Te87); or c) both. As noted in our Progress Report last year, a large carcinogenesis experiment was designed and set up to distinguish among these possibilities (Table 1). The final analyses of these data are now complete, and the manuscript is to be submitted within a few weeks (Do91; Appendix IV).

The keys to this experimental design are: a) that each rat in control groups $\mathrm{C}, \mathrm{CE}$, and $\mathrm{CM}$ received the same total number of intact thyroid clonogens as each rat in their respective paired irradiated cell recipient experimental groups R, RE and RM, and b) that within a given group, every grafted clonogen number was transplanted in an animal that received the same total clonogen numbers. This design ruled out any differences in grafted cell number-dependent neoplastic promotion-progressic - by TSH which might have occured in the previous experiments in which recipients of low cell numbers per graft had fewer total grafted cells than those with high cell numbers per graft. Groups CE and RE were designed to show any systemic tumor-suppressive effect. Groups CM and RM were included to investigate the possibility of promotion-progression suppressing local cell-cell interactions. 
Table 1: Design of experiment to investigate hormonal and local inhibition of promotion/progression in radiation-initiated thyroid clonogens.

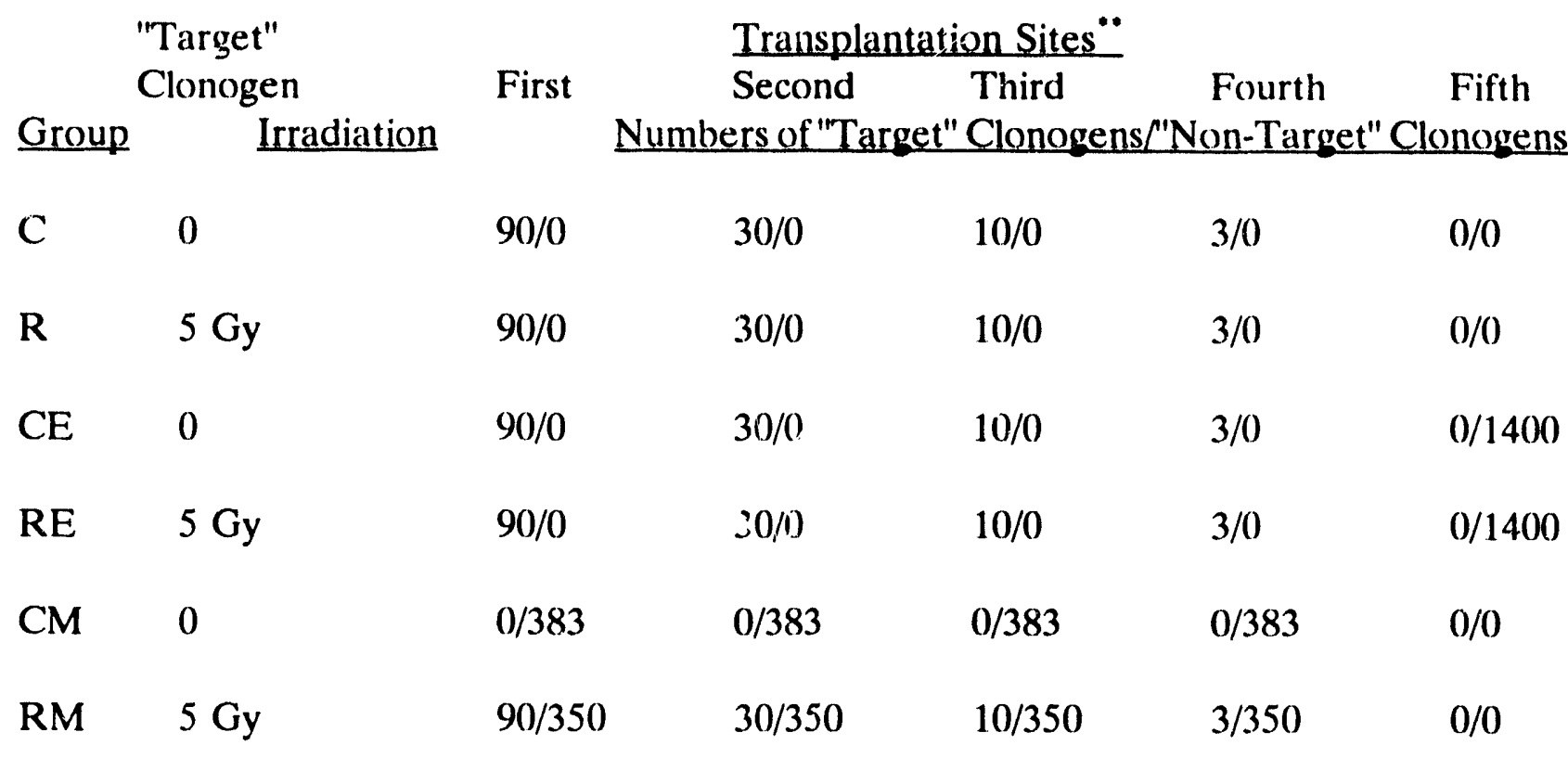

"Target" clonogens are irradiated clonogens and their paired unirradiated clonogen controls. "Non-target" clonogens are unirradiated clonogens added to test hormonal and local inhibition of promotion/progression in "target" clonogens.

"*Transplantation sites were rotated during grafting such that, for example, the interscapular pad might be the first site in rat \#1, the second in rat \#2, the third in rat \#3, etc. The experiment was set up in 20 replicate runs to accumulate 100 graft sites for each combination of tiansplanted clonogens.

Briefly, the results of this experiment are:

1) As found previously, the incidence of radiogenic initiation is very high. Estirnates based on the frequency of cancer in the graft sites that received three irradiated or control clonogens in groups $\mathrm{R}$ and $\mathrm{C}$ indicate that one of five clonogens was initiated. As we have suggested (C190, C191; Appendix V), such frequencies strongly suggest that initiation is a generic term covering any event, genetic or epigenetic, which alters the probability that a cell or its progeny wil! give rise to cancer. Such events may include alterations in growth regulatory genes, but perhaps more frequently are radiation induced non-mutational changes (C190, C191; Appendix V). Among the latter may be the induction of SOS-like error-prone repair, and alterations in DNA methylation which influence gene function.

2) As previously found (Mu84, Wa88), 5 Gy low LET radiation to the clonogens before 
transplantation significantly increased the carcinomas per $10^{4}$ graft weeks at each cell dose in group $\mathrm{R}$ as compared to those in the unirradiated cells in group $\mathrm{C}$, and similarly in group $\mathrm{RE}$ as compared to group $\mathrm{CE}$.

3) There were no detectable grafted cell number-related differences in carcinoma incidence or latency or in graft size in groups RM or CM due to the high cancer incidence from the large number of unirradiated clonogens in each graft site.

4) More carcinomas occurred per $10^{4}$ graft weeks at each cell dose in group $R$ than in group RE. Differences in tumor incidence per graft week between groups $C$ and $C E$ were not significant. Irrespective of histopathology, the diameters of the grafts per hundred graft weeks increased directly and significantly with grafted clonogen numbers in groups R, C, RE and $C E$. At each cell dose, graft diameters in R exceeded those in C, and those in RE those in CE. The serum TSH titers in groups RE and CE, though significantly greater than normal, were significantly less than in groups $\mathrm{R}$ and $\mathrm{C}$.

The differences in tumor incidence and graft diameters among the above experimental groups are thus very likely due to less intense promotion-progression in groups $\mathrm{RE}$ and $\mathrm{CE}$ than in groups $\mathrm{R}$ and $\mathrm{C}$ as a result of thyroid hormone release from FU formed by the large number of unirradiated grafted cells in the fifth graft sites in RE and CE. We conclude that there can be a grafted cell number-related suppression of neoplastic promotion-progression mediated by systemic inhibition of TSH secretion by thyroid hormone release from FU that develop in the grafts even in the presence of iodine deficiency.

Suppression of promotion-progression by systemic inhibition of TSH secretion does not, however, account for the relatively lower incidence of carcinomas per graft-week per grafted cell in the high cell dose sites as compared to the low cell dose sites of groups $\mathrm{R}$ and RE, e.g. a four- to six-fold difference in carcinomas versis a 30 -fold difference in grafted cell number. There are two inherent sources of uncertainty which we believe account for these differences in observed cancer incidences. First, during transplantation, the intact clonogens may be assumed to be randomly distributed in the inoculum. Hence, at a given mean grafted clonogen number per site, there will be a normal distribution of clonogen numbers per site surrounding the mean. At low cell numbers per site, this will include some sites with no clonogens, and hence no possibility for tumor formation. Conversely, there will be some sites with more than the mean number of clonogens and thus a slightly higher probability of cancer formation than the mean. More importantly, the cancer incidence was conservatively derived; i.e. only one carcinoma was scored per graft site. In those sites with more than one grafted clonogen, more than one carcinoma might arise; indeed in those sites grafted with many irradiated clonogens, the probability that more than one carcinoma arose or would have arisen is high.

A mathematical correction (Ha79) based on the assumption of normal distributions both of the clonogens in the inocula during transplantation and of the progression of initiated clonogens to cancer can be applied to the data to estimate the true number of 
carcinomas from the observed number. Thus far, such calculations have been made only to correct for multiple tumors per graft site. When the data are so corrected, the cell dose dependent differences in the cancer incidences per grafted clonogen within a given experimental group are no longer significant. We thus conclude that our results do not support the hypothesis that local grafted cell number dependent cell-cell interactions which suppress neoplastic promotion-progression exist in this system (Do91; Appendix IV).

Closing comments: We believe that the concept that the thyroid contains TSHresponsive clonogens that are the ultimate source of new cells as weil as the progenitor cells in carcinogenesis, and that neoplastic initiation is a frequent event in these cells, will alter the general understanding of both normal thyroid physiology and of cancer formation. As noted above, new cell culture and cell sorting methodologies have been introduced and are under test for both mammary and thyroid cells. We believe these may be very useful for the further chracterization of the thyroid clonogens and their role in thyroid neoplasia. We believe that continuing short term studies of clonogen physiology in parallel with long-term carcinogenesis studies is an efficient way to proceed, and that the presence of a second parallel mammary clonogen system in which major observations can be cross-checked for broader applicability adds credibility to our findings. The techniques developed in or adapted to these laboratories represent a unique armamentarium for the study of thyroid cancer development in particular, and of radiogenic carcinogenesis in general.

Personnel and effort 1/1/91-5/31/91: During this period, the P.I. spent 25\% of his time on this project, and Professor Gould has been available as needed. Mrs. Jane Barnes, laboratory manager, has spent $60 \%$ of her time in planning of the logistics of this project, in the day-to-day coordination and in specialized technical work and technique development. Mrs. Joan Mitchen, a highly skilled technician, has spent $80-85 \%$ of her time on the project helping with all aspects of the technology. She has learned the process of entering data into computer spread sheets for analysis, and has played an essential role in this project. Dr. Kevin Groch, who received his $\mathrm{PhD}$ in November 1990, is currently doing postdoctoral work in Professor Gould's laboratory, and has thus been available to complete the manuscripts on thyroid clonogen kinetics and function during goitrogenesis. Dr. Frederick Domann just completed his $\mathrm{PhD}$. He has spent considerably more than the $50 \%$ of his time suggested by his stipend level on this project during this period. Mr. Jinchuan Zhao, an excellent young student, joined our laboratory in September of 1990 to work toward the $\mathrm{PhD}$, and is spending $50 \%$ of his time on the project.

The principal investigator believes that we have complied with the requirements of the grant in all respects. 


\section{References:}

Note: $\quad$ References marked with an asterisk are included as Appendices I-V.

C180 Clifton, K.H. Quantitative studies of the radiobiology of hormone responsive normal cell populations. In: Radiation Biology in Cancer Research, 32nd M.D. Anderson Symposium on Fundamental Cancer Research, R.E. Meyn and H.R. Withers, eds., Raven Press: New York, pp. 501-513, 1980.

Cl89 Clifton, K.H., Kamiya, K., Groch, K.M. and Domann, F.E. Quantitative studies of rat mammary and thyroid clonogens, the presumptive cancer progenitor cells. In: Cell Transformation and Radiation-induced Cancer in Man, pp. 135-145. K.H. Chadwick, C. Seymour and B. Barnhart, eds. Bristoi, U.K.: IOP Publishing Ltd., 1989.

Cl90 Clifton, K.H. The clonogenic cells of the rat mammary and thyroid glands: Their biology, frequency of initiation and promotion/progression to cancer. In: Mathematical Modeling. Statistical Issues in Cancer Risk Assessment, pp. 1-21. S. Moolgavkar, ed. Boston: Birkhauser Inc., 1989.

'Cl91 Clifton, K.H., Domann, F.E. and Groch, K.M. On the cells of origin of radiogenic thyroid cancer: New studies based on an old idea. J. Radiat. Res. (Japan) (in press). (Appendix V)

Co90 Committee on the Biolog,ical Effects of Ionizing Radiation. Health Effects of Exposure to Low Lev $1 / s$ of Ionizing Radiation (BEIR V). Washington: National Academy Press, 1990.

'Do90 Domann, F.E., Mitchen, J.M. and Clifton, K.H. Restoration of thyroid function following total thyroidectomy and quantitative thyroid cell transplantation. Endocrinology 127:2673-2678, 1990. (Appendix I)

'Do91 Domann, F.E., Gould, M.N. and Clifton, K.H. In vivo assessment of the radiogenic cancer initiation frequency per clonogenic rat epithelial cell. (Submitted) (Appendix IV)

"Gr91a Groch, K.M. and Clifton, K.H. The plateau phase rat goiter contains a subpopulation of TSH-responsive epithelial cells capable of clonal proliferation following transplantation. Acta Endocrinologica (in press), 1991. 
(Appendix II)

"Gr90b Groch, K.M. and Clifton, K.H. The effects of goitrogenesis, involution, and goitrogenic rechallenge on the clonogenic cell content of the rat thyroid. Submitted. (Appendix III)

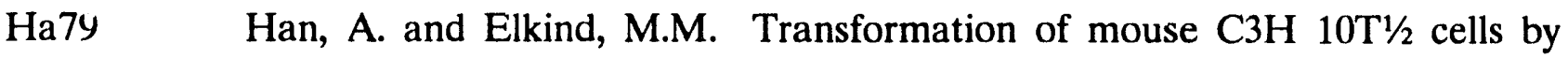
single and fractionated doses of $\mathrm{x}$-rays and fission spectrum neutrons. Cancer Res. 39:123-130, 1979.

Mu84 Mulcahy, R.T., Gould, M.N. and Clifton, K.H. Radiogenic initiation of thyroid cancer: A common cellular event. Int. J. Radiat. Biol. 45:419-426, 1984.

Te87 Terzaghi-Howe, M. Inhibition of carcinogen-altered rat tracheal epithelial cell proliferation by normal epithelial cells in vivo. Carcinogenesis 8:145-150, 1987.

Wa88 Watanabe, H., Tanner, M.A., Domann, F.E., Gould, M.N. and Clifton, K.H. Inhibition of carcinoma formation and of vascular invasion in grafts of radiation-initiated thyroid clonogens by unirradiated thyroid cells. Carcinogenesis 9:1329-1335, 1988. 

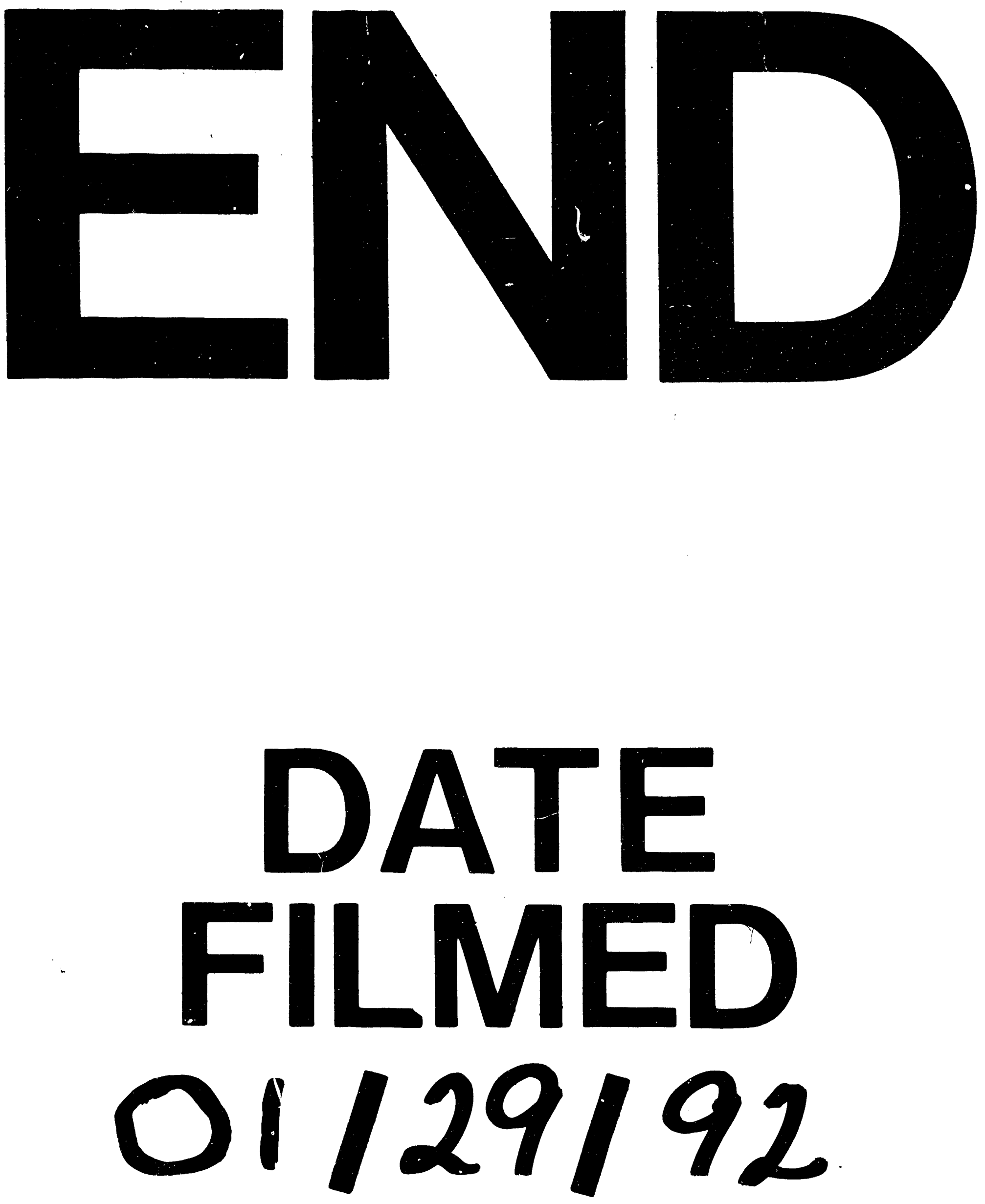

i 